\title{
A NOTE ON THE MOTION OF SURFACES
}

\author{
Robert I. McLachlan And Harvey Segur
}

June 22, 1993

\begin{abstract}
We study the motion of surfaces in an intrinsic formulation in which the surface is described by its metric and curvature tensors. The evolution equations for the six quantities contained in these tensors are reduced in number in two cases: (i) for arbitrary surfaces, we use principal coordinates to obtain two equations for the two principal curvatures, highlighting the similarity with the equations of motion of a plane curve; and (ii) for surfaces with spatially constant negative curvature, we use parameterization by Tchebyshev nets to reduce to a single evolution equation. We also obtain necessary and sufficient conditions for a surface to maintain spatially constant negative curvature as it moves. One choice for the surface's normal motion leads to the modified-Korteweg de Vries equation, the appearance of which is explained by connections to the AKNS hierarchy and the motion of space curves.
\end{abstract}

\section{InTRODUCTION AND EQUATIONS OF MOTION}

Studies of the motion of surfaces and interfaces occur in several scientific disciplines, including geometry $[8,10,17]$, water waves [20], crystal growth $[11,13]$, combustion [4], and gas dynamics [18]. Pelcé [16] collected and reprinted an interesting collection of original articles in some of these areas. To simplify the analysis, these studies often consider only two-dimensional motion, thus reducing the problem to the motion of a curve [6], but this is only a special case of the usual situation.

To study the evolution of two-dimensional surfaces arbitrarily embedded in three dimensions, a natural approach is to describe the surface purely intrinsically, that is, in terms of the surface's metric and curvature tensors. In these variables, the equations of motion for the surface take the form of six coupled nonlinear evolution equations for the six independent components of the two tensors. These equations were first written down by Brower et al. [3], and later by Nakayama \& Wadati [15].

Unfortunately, these equations are highly redundant. To see this, observe that if a surface that can be represented at a fixed time in the form $z=z(x, y)$, then its motion can be described by a single evolution equation, for $\partial z / \partial t$. The purpose of this paper is to simplify the evolution equations obtained in [3] and [15], by reducing the number of unknowns. We obtain two simplifications, by choosing two different parameterizations of the surface.

The first parameterization, in $\S 2$, is general: we parameterize a surface by its principal lines (i.e., lines tangent to the directions of principal curvature). This

Typeset by $\mathcal{A} \mathcal{M S}-\mathrm{TEX}_{\mathrm{E}}$ 
reduces the system to four coupled equations, and, with a particular choice of parameterization along the principal lines, to two equations for the two curvatures. If the surface happens to be a cylinder above a curve in a plane, and if the motion is two-dimensional, then one curvature vanishes and the equations reduce to the single equation obtained in $[3,14]$ for the evolution of the curvature of a plane curve.

The second parameterization, in $\S 3$, is more specialized: surfaces of constant negative curvature have an elegant parameterization by Tchebyshev nets. For surfaces that maintain constant negative curvature as they move, their motion is specified by a single evolution equation for the angle between the coordinate lines. As an example, we show that one choice for the normal velocity of the surface leads to the modified Korteweg-de Vries equation for the surface's evolution.

For either set of coordinates discussed here, the key idea is to force the coordinate lines to maintain their defining property as the surface evolves, by adding an appropriate tangential velocity to the evolution.

The first step is to derive the six coupled equations for the motion of a surface moving in $\mathbb{R}^{3}$. Local coordinates on the surface are $\left(x_{1}, x_{2}\right)=\mathbf{x}$, time is $t$, and the position (in $\mathbb{R}^{3}$ ) of a point on the surface is $\mathbf{r}(\mathbf{x}, t)$. Later $\dot{\mathbf{r}}(=\partial \mathbf{r} / \partial t)$ will be specified, so one can think of $\mathbf{x}$ as a Lagrangian particle label on the surface. Define two tangent vectors and one normal vector:

$$
\tau_{\mu}=\mathbf{r}_{, \mu} \quad \mathbf{n}=\frac{\tau_{1} \times \tau_{2}}{\left|\tau_{1} \times \tau_{2}\right|}
$$

where greek indices range over $\mu=1,2$ with,$\mu=\frac{\partial}{\partial x_{\mu}}$. (Both the notation used here and the preliminary development follow Spivak [19, Chapter 2].) Note that $\mathbf{n}$ is a unit vector but the $\boldsymbol{\tau}_{\mu}$ are not necessarily unit vectors. We have the metric $g_{\mu \nu}$ and curvature $h_{\mu \nu}$ tensors (which define the first and second fundamental forms $g_{\mu \nu} d x_{\mu} d x_{\nu}$ and $h_{\mu \nu} d x_{\mu} d x_{\nu}$, respectively):

$$
g_{\mu \nu}=\boldsymbol{\tau}_{\mu} \cdot \boldsymbol{\tau}_{\nu}, \quad h_{\mu \nu}=\mathbf{n} \cdot \tau_{\mu, \nu}=\mathbf{n} \cdot \mathbf{r}_{, \mu \nu}
$$

As one moves along the surface (at a fixed time), the tangent and normal vectors change according to the Gauss-Weingarten equations,

$$
\begin{aligned}
\boldsymbol{\tau}_{\mu, \nu} & =\boldsymbol{\tau}_{\lambda} \Gamma_{\mu \nu}^{\lambda}+\mathbf{n} h_{\mu \nu} \\
\mathbf{n}_{, \nu} & =-\boldsymbol{\tau}_{\lambda} g^{\lambda \mu} h_{\mu \nu}
\end{aligned}
$$

where $\Gamma_{\mu \nu}^{\lambda}$ are the Christoffel symbols of the second kind defined by the metric $g_{\mu \nu}$, and $g^{\mu \nu}=\left(g_{\mu \nu}\right)^{-1}$ as usual. Repeated indices are summed on unless otherwise noted.

The six quantities in (1.1) are not all independent. They are related by three consistency conditions for the PDE's (1.2) - the Gauss-Codazzi equations:

$$
\begin{aligned}
R_{1212} & =\operatorname{det} h \\
h_{\nu \lambda ; \mu} & =h_{\mu \lambda ; \nu}
\end{aligned}
$$

where $; \mu$ denotes covariant derivative and $R_{1212}$ is the nontrivial element of the Riemann tensor. Note that $(1.3 \mathrm{~b})$ gives independent information only for 
$(\nu, \lambda, \mu)=(1,1,2)$ or $(2,2,1)$. Of the three remaining degrees of freedom, two are due to the arbitrary parameterization, leaving one - which we expect, since the surface could be written, e.g., $\mathbf{r}=(\mathbf{x}, z(\mathbf{x}), t)$.

Therefore one might ask: What is to be gained by going to an "intrinsic" (i.e. $g_{\mu \nu}, h_{\mu \nu}$ ) representation of the surface when eqs. (1.3) cannot usually be solved explicitly? Any surface motion can after all be formulated locally as $\dot{z}=f(\mathbf{x}, t)$. Such an approach fails if the surface becomes vertical - an apparently artificial constraint-but the intrinsic formulation can also suffer from artificial coordinate singularities. One advantage is that in some applications, the motion of the surface normal to itself is given naturally in terms of the local curvatures $[3,17]$ which are the eigenvalues of $g^{\mu \lambda} h_{\lambda \nu}$; secondly, such an approach has been successful in the study of the motion of curves, as we now discuss.

Hasimoto [9] showed that in the self-induction approximation, the motion of a $3 \mathrm{D}$ vortex filament (which moves in the direction of its local binormal vector with speed equal to its local curvature) can be reduced to the nonlinear Schrödinger equation. Lamb [12] extended this to more general motions of curves, and found motions obeying the sine-Gordon and modified Korteweg-de Vries equations. All three of these equations are completely integrable [1], and Goldstein and Petrich [7] showed how to specify infinitely many two-dimensional motions of curves in terms of integrable evolution equations. The occurrence of such integrable equations was shown in [14] to be due to the fact that the Serret-Frenet equations, $(\mathbf{t}=\boldsymbol{\tau} /|\boldsymbol{\tau}|, s=$ arclength $)$

$$
\left(\begin{array}{l}
\mathbf{t}, s \\
\mathbf{n}_{, s}
\end{array}\right)=\left(\begin{array}{cc}
0 & \kappa \\
-\kappa & 0
\end{array}\right)\left(\begin{array}{l}
\mathbf{t} \\
\mathbf{n}
\end{array}\right)
$$

which are the analog of (1.2) for plane curves, are equivalent to the AKNS scattering problem [1] at zero eigenvalue. Thus evolutions $\left(\begin{array}{c}\dot{\mathbf{t}} \\ \dot{\mathbf{n}}\end{array}\right)$ can be specified which give rise to integrable equations of the AKNS hierarchy.

The equations for surfaces, (1.2), are more complicated than those for curves, (1.4), for two main reasons: (i) $\mathbf{t}$ in (1.33) is a unit vector, whereas the $\tau_{\mu}$ in (1.2) are not; and (ii) the coordinates, $x_{i}$, in (1.2) need not measure arclength. If we relax these two requirements in (1.4), then they become

$$
\left(\begin{array}{l}
\boldsymbol{\tau}_{, x} \\
\mathbf{n}_{, x}
\end{array}\right)=\left(\begin{array}{cc}
g_{, x} / 2 g & h \\
-h / g & 0
\end{array}\right)\left(\begin{array}{l}
\boldsymbol{\tau} \\
\mathbf{n}
\end{array}\right)
$$

which are equivalent to (1.2) when $\mu=1$ only.

There is another interesting connection between curves and surfaces which we note briefly. In [14] it is shown that the Serret-Frenet equations for space curves also reduce to the AKNS scattering problem at zero eigenvalue (i.e., they take the form (1.4) in appropriate variables). Considering a moving space curve as sweeping out a surface, we see that the equations analogous to Serret-Frenet for the surface, the Gauss-Weingarten equations (1.2), must also contain the AKNS scattering problem (in appropriate variables). This point of view explains the occurrence of integrable equations from the AKNS hierarchy in the equations defining particular surfaces, such as the sine-Gordon equation for surfaces of constant negative curvature. 
Motion of a surface can be prescribed in the form

$$
\dot{\mathbf{r}}=U \mathbf{n}+V^{\mu} \boldsymbol{\tau}_{\mu}
$$

where $U$ and $V^{\mu}$ can be specified arbitrarily. This implies (differentiate $\boldsymbol{\tau}_{\mu}=\mathbf{r}_{, \mu}$ and $\mathbf{n} \cdot \boldsymbol{\tau}_{\mu}=0$ and use $\left.(1.2)\right)$ :

$$
\begin{aligned}
\dot{\boldsymbol{\tau}}_{\mu} & =\left(U_{, \mu}+V^{\nu} h_{\mu \nu}\right) \mathbf{n}+\left(-U h_{\mu}^{\lambda}+V_{; \mu}^{\lambda}\right) \boldsymbol{\tau}_{\lambda} \\
\dot{\mathbf{n}} & =-g^{\lambda \mu}\left(U_{, \mu}+V^{\nu} h_{\mu \nu}\right) \boldsymbol{\tau}_{\lambda}
\end{aligned}
$$

Now intrinsic evolution equations for $\dot{g}_{\mu \nu}, \dot{h}_{\mu \nu}$ can be derived in two ways. The first is to differentiate (1.1) with respect to $t$ and to eliminate $\boldsymbol{\tau}_{\mu}$ and $\mathbf{n}$ using $(1.2,1.3)$. This was first done by Brower [3] for $V^{\mu}=0$ and by Nakayama et al. [15] for $V^{\mu} \neq 0$. There is also an "intrinsic" derivation, namely requiring compatibility of the PDE's (1.2) and (1.6). This gives equations which must be solved for $\dot{g}_{\mu \nu}$ and $\dot{h}_{\mu \nu}$, and the result is (of course) the same:

$$
\begin{aligned}
& \dot{g}_{\mu \nu}=-2 h_{\mu \nu} U+V_{\mu ; \nu}+V_{\nu ; \mu} \\
& \dot{h}_{\mu \nu}=U_{; \mu \nu}-h_{\mu \lambda} h_{\nu}^{\lambda} U+h_{\mu \lambda} V_{; \nu}^{\lambda}+h_{\nu \lambda} V_{; \mu}^{\lambda}+V^{\lambda} h_{\mu \lambda ; \nu}
\end{aligned}
$$

Notice that $V^{\mu}$ does not affect the actual shape of the surface. The easiest way to see this is to note that the evolution in (1.5) is linear in $U, V^{\mu}$, so we can consider $U=0$; then the $V^{\mu}$ velocities just push particles around on a motionless surface. Notice also that if a coordinate system with some particular defining property is chosen initially, that property will be destroyed under general motion of the particles on the surface. Therefore we will use the tangential velocities $V^{\mu}$ to "push" the coordinates back, so that they maintain their defining property as the surface moves. (In the next sections we show how to do this in some special cases.) Nakayama et al. [15] in addition allowed the coordinates to evolve on the surface, but this does not give any extra generality.

\section{Principal COORDinates}

The principal curvatures $\left(\kappa_{1}, \kappa_{2}\right)$ and principal directions of a surface are the eigenvalues and eigenvectors of $g^{\mu \lambda} h_{\lambda \nu}$. If the coordinate lines are chosen to be tangent to the principal directions at each point on the surface, then $g_{\mu \nu}$ and $h_{\mu \nu}$ are diagonal, and we have principal coordinates. These can be defined globally on any surface, and are smooth except where $\kappa_{1}=\kappa_{2}$-umbilic points. (See [19, p. 288] for more information about umbilic points; they are generally isolated.) We define $g_{1}, g_{2}, \kappa_{1}$, and $\kappa_{2}$ by

$$
g_{\mu \nu}=\left(\begin{array}{cc}
e^{g_{1}} & 0 \\
0 & e^{g_{2}}
\end{array}\right), \quad h_{\mu \nu}=\left(\begin{array}{cc}
e^{g_{1}} \kappa_{1} & 0 \\
0 & e^{g_{2}} \kappa_{2}
\end{array}\right) .
$$

Note that $g_{i}$ and $\kappa_{i}$ do not form covariant vectors - they are just functions appearing in the 2-tensors $g_{\mu \nu}$ and $h_{\mu \nu}$.

In terms of these new variables, the Gauss-Codazzi equations (1.3b) now read

$$
g_{1,2}=\frac{2 \kappa_{1,2}}{\kappa_{2}-\kappa_{1}}, \quad g_{2,1}=-\frac{2 \kappa_{2,1}}{\kappa_{2}-\kappa_{1}}
$$


which we will use to simplify the subsequent equations. For completeness, we also give the Gauss-Weingarten formulae in these variables:

$$
\begin{gathered}
\frac{\partial}{\partial x_{1}}\left(\begin{array}{c}
\boldsymbol{\tau}_{1} \\
\boldsymbol{\tau}_{2} \\
\mathbf{n}
\end{array}\right)=\left(\begin{array}{ccc}
\frac{1}{2} g_{1,1} & -\frac{1}{2} g_{1,2} e^{g_{1}-g_{2}} & e^{g_{1}} \kappa_{1} \\
\frac{1}{2} g_{1,2} & \frac{1}{2} g_{2,1} & 0 \\
-\kappa_{1} & 0 & 0
\end{array}\right)\left(\begin{array}{c}
\boldsymbol{\tau}_{1} \\
\boldsymbol{\tau}_{2} \\
\mathbf{n}
\end{array}\right) \\
\frac{\partial}{\partial x_{2}}\left(\begin{array}{c}
\boldsymbol{\tau}_{1} \\
\boldsymbol{\tau}_{2} \\
\mathbf{n}
\end{array}\right)=\left(\begin{array}{ccc}
\frac{1}{2} g_{1,2} & \frac{1}{2} g_{2,1} & 0 \\
-\frac{1}{2} g_{2,1} e^{g_{2}-g_{1}} & \frac{1}{2} g_{2,2} & e^{g_{2}} \kappa_{2} \\
0 & -\kappa_{2} & 0
\end{array}\right)\left(\begin{array}{c}
\boldsymbol{\tau}_{1} \\
\boldsymbol{\tau}_{2} \\
\mathbf{n}
\end{array}\right)
\end{gathered}
$$

As the surface moves, the coordinate lines will not remain principal coordinates unless $\dot{g}_{12}=\dot{h}_{12}=0$. (This is where we begin to specify $V^{\mu}$ in order to maintain the desired coordinate system.) This leads to two equations in $V_{, 2}^{1}$ and $V_{, 1}^{2}$ whose solution is

$$
V_{, 2}^{1}=e^{-g_{1}} f, \quad V_{, 1}^{2}=-e^{-g_{2}} f
$$

where

$$
f=\frac{\left(U_{, 2} \kappa_{2}\right)_{, 1}-\left(U_{, 1} \kappa_{1}\right)_{, 2}}{\left(\kappa_{2}-\kappa_{1}\right)^{2}} .
$$

With these choices, (1.7) reduces to four coupled equations:

$$
\begin{aligned}
& \dot{g}_{i}=-2 \kappa_{i} U+V^{j} g_{i, j}+2 V_{, i}^{i} \\
& \dot{\kappa}_{i}=\kappa_{i}^{2} U+V^{j} \kappa_{i, j}+e^{-g_{i} / 2}\left(e^{-g_{i} / 2} U_{, i}\right)_{, i}+e^{-g_{i^{\prime}}} g_{i, i^{\prime}} U_{, i^{\prime}} / 2
\end{aligned}
$$

$\left(\left(i, i^{\prime}\right)=(1,2)\right.$ or $(2,1)$; no sum on $\left.i, i^{\prime}\right)$. These may be written as

$$
\begin{aligned}
& \frac{D g_{i}}{D t}-2 V_{, i}^{i}=-2 \kappa_{i} U \\
& \frac{D \kappa_{i}}{D t}=\kappa_{i}^{2} U+U_{, s_{i} s_{i}}+e^{-g_{i^{\prime}}} g_{i, i^{\prime}} U_{, i^{\prime}} / 2
\end{aligned}
$$

where $D / D t$ is total derivative in the direction $-V^{j} \partial_{j}$ (i.e. the right hand side of (2.5) gives the evolution at "unpushed" particle labels) and $s_{i}$ is arclength along $x_{i}$, (i.e. $\left.s_{1}=\int^{x_{1}} \exp \left(\frac{1}{2} g_{1}\left(\dot{x}_{1}, x_{2}\right)\right) d \dot{x}_{1}\right)$.

As a special case of these equations, we note that if $\kappa_{2}=\partial_{2}=0$ so that the surface only depends on $x_{1}$, then $f=0$ in (2.3), we can take $V^{2}=0$, $V^{1}=W\left(x_{1}\right) \exp \left(-\frac{1}{2} g_{1}\right)$ say, and we recover the equations for the motion of a plane curve [14]:

$$
\dot{g}=2 W_{, s}-2 \kappa U \quad \dot{\kappa}=\kappa^{2} U+U_{, s s}+W \kappa, s
$$

Now we return to the general case, to reduce (2.4) to two coupled equations for the evolution of the two curvatures, $\kappa_{1}$ and $\kappa_{2}$. These two equations, along with their compatibility condition, (1.3a), seem to be the simplest intrinsic formulation possible for a general surface.

In (2.3), the equations for $V^{\mu}$, there is still the freedom to specify two arbitrary functions, each of one variable. We can use this freedom to specify $g_{i}$, and hence the metric, completely. Here is one way to specify the metric: require

$$
g_{1}\left(x_{1}, 0, t\right)=g_{2}\left(0, x_{2}, t\right)=0 \quad \forall t,
$$


so that $g_{11}=g_{22}=1$ along these two coordinate lines, which are both parameterized by arclength (see Figure 1). Let $v^{\mu}$ be two functions of integration in (2.3), i.e.,

$$
\begin{aligned}
& V^{1}\left(x_{1}, x_{2}\right)=+\int_{0}^{x_{2}} \exp \left(-g_{1}\left(x_{1}, x_{2}\right)\right) f\left(x_{1}, \dot{x}_{2}\right) d \dot{x}_{2}+v^{1}\left(x_{1}\right) \\
& V^{2}\left(x_{1}, x_{2}\right)=-\int_{0}^{x_{1}} \exp \left(-g_{2}\left(\dot{x}_{1}, x_{2}\right)\right) f\left(\dot{x}_{1}, x_{2}\right) d \dot{x}_{1}+v^{2}\left(x_{2}\right)
\end{aligned}
$$

where we have suppressed the explicit $t$ dependence. Then, from (2.4a) and choosing $v_{i}(0)=0$, enforcing (2.6) requires

$$
v_{i}^{\prime}=\left.\left(\kappa_{i} U-\frac{1}{2} g_{i, i^{\prime}} V^{i^{\prime}}\right)\right|_{x_{i^{\prime}}=0}
$$

Now (2.1) can be integrated to define $g_{i}$ completely:

$$
g_{1}=\int_{0}^{x_{2}} \frac{2 \kappa_{1,2}}{\kappa_{2}-\kappa_{1}} d \dot{x}_{2}, \quad g_{2}=-\int_{0}^{x_{1}} \frac{2 \kappa_{2,1}}{\kappa_{2}-\kappa_{1}} d \dot{x}_{1} .
$$

Substituting these into (2.4b) yields two coupled equations for the evolution of the two curvatures, as desired.

In the $\kappa_{2}=\partial_{2}=0$ case, $v_{1}^{\prime}=\kappa_{1} U$ and in this gauge, $x_{1}$ is arclength, so we get $W=\int \kappa_{1} U d s$ as expected.

\section{Surfaces of Constant negative Curvature}

From the wealth of special surfaces and coordinate systems available, we consider a particular choice for which the equations of motion take a particularly simple form. At every point on a surface whose Gaussian (or total) curvature $K$ is negative, the curvature tensor has two null vectors (i.e. vectors $v^{\mu}$ which annihilate $h_{\mu \nu}$, so $v^{\mu} v^{\nu} h_{\mu \nu}=0$; see [19, p. 70]). An asymptotic line is a smooth curve on the surface whose tangent vector coincides with one of these null vectors at each point along the curve. If the Gaussian curvature is constant (in space) and negative, then there are two sets of asymptotic lines, and they form a Tchebyshev net, i.e., they can be parameterized by arclength everywhere [19, p. 368]. With these as coordinate lines, the metric and curvature tensors take the form

$$
g_{\mu \nu}=\left(\begin{array}{cc}
1 & \cos \omega \\
\cos \omega & 1
\end{array}\right), \quad h_{\mu \nu}=\left(\begin{array}{cc}
0 & h_{12} \\
h_{12} & 0
\end{array}\right)
$$

where $\omega$ measures the angle between the coordinate lines. Furthermore, the Gauss-Codazzi equations become:

$$
\begin{aligned}
h_{12} & =\sqrt{-K} \sin \omega, \\
\omega_{, 12} & =-K \sin \omega,
\end{aligned}
$$

i.e., solutions of the sine-Gordon equation are in a local 1-1 correspondence with such surfaces. Unfortunately such coordinates may be only local [19, p. 373]. 
The Gauss-Weingarten equations (1.2) also take the simple form

$$
\begin{aligned}
& T_{, 1}=\left(\begin{array}{ccc}
\omega_{, 1} \cot \omega & -\omega_{, 1} \csc \omega & 0 \\
0 & 0 & \sqrt{-K} \sin \omega \\
\sqrt{-K} \cot \omega & -\sqrt{-K} \csc \omega & 0
\end{array}\right) T \\
& =\left(\begin{array}{ccc}
-\left(\ln \left(\kappa_{1}-\kappa_{2}\right)\right)_{1} & -\left(\ln \left(\kappa_{1}+\kappa_{2}\right)\right)_{1} & 0 \\
0 & 0 & -\kappa_{1} \kappa_{2} /\left(\kappa_{1}-\kappa_{2}\right) \\
\kappa_{1}+\kappa_{2} & -\left(\kappa_{1}-\kappa_{2}\right) & 0
\end{array}\right) T
\end{aligned}
$$

and similarly for $T_{, 2}$, where

$$
T=\left(\begin{array}{c}
\boldsymbol{\tau}_{1} \\
\boldsymbol{\tau}_{2} \\
\mathbf{n}
\end{array}\right)
$$

and $\kappa_{1}, \kappa_{2}$ are the principal curvatures $\sqrt{-K}(\cot \omega \pm \csc \omega)$.

The first step is to find those normal velocities $U$ that keep the Gaussian curvature constant in space (but not necessarily in time). Substitute (3.1) into (1.7), and require both that the form of (3.1) be preserved (i.e., $\dot{g}_{11}=\dot{g}_{22}=$ $\dot{h}_{11}=\dot{h}_{22}=0$ ) and consistency (so that $\dot{\omega}$ from (1.7a) equals that from $(1.7 \mathrm{~b})$ ). One finds in this way that $U$ must satisfy a linear inhomogeneous equation:

$$
U_{, 12}=-K U \cos \omega+(\sin \omega) \frac{d}{d t} \sqrt{-K}
$$

and that the tangential components of velocity must satisfy:

$$
V_{, i^{\prime}}^{i}=-\left(K U \sin \omega+\omega_{, i^{\prime}}\left((-1)^{i^{\prime}} U_{, i^{\prime}} \cos \omega+U_{, i}\right)+U_{, i^{\prime} i^{\prime}} \sin \omega\right) / 2 \sqrt{-K} \sin ^{2} \omega
$$

where $i, i^{\prime}=(1,2)$ or $(2,1)$, and there is no sum on $i^{\prime}$. There are no other constraints. As mentioned earlier, only the normal component of velocity actually moves the surface, so we have established the following

Proposition: Suppose that a surface whose Gaussian curvature $K$ is constant in space and negative moves according to (1.5). It will maintain (spatially) constant negative curvature if and only if $U$ satisfies (3.4).

Note that if $\dot{K}=0$, then (3.4) is just the linearization of the sine-Gordon equation, (3.2b), so its translational symmetry immediately gives two solutions of (3.4): $U=\omega_{, 1}$ and $U=\omega_{, 2}$. With the velocity components chosen to satisfy (3.4) and (3.5), the evolution of $\omega$ is given by

$$
\frac{D \omega}{D t}+\left(V_{, 2}^{1}+V_{, 1}^{2}\right) \sin \omega=2 \sqrt{-K} U
$$

where $D / D t$ was defined below (2.5).

As an example, take $\dot{K}=0$ and $U=\omega, 1\left(=\omega, s_{1}\right)$. Eqs. (3.5) can be integrated to find

$$
V^{1}=\left((\omega, 1)^{2}+2 \omega_{, 11} \cot \omega\right) / 4 \sqrt{-K}, \quad V^{2}=-\omega, 11 \csc \omega / 2 \sqrt{-K}
$$

SO

$$
\dot{\omega}=\frac{1}{4}\left(6 \sqrt{-K} \omega_{, 1}+\left((\omega, 1)^{3}+2 \omega_{, 111}\right) / \sqrt{-K}\right)
$$


which is the modified Korteweg-de Vries equation in the variable $\omega_{, 1}$. The occurrence of an equation from the AKNS hierarchy is not surprising, for the following reason. Let $\omega\left(x_{1}, 0\right)$ be an arbitrary function. It can be integrated in $x_{2}$ under (3.2b) to sweep out a surface of constant negative curvature, or (because (3.7) does not depend explicitly on $x_{2}$ ) it can be integrated in $t$ under (3.7). These two AKNS evolution equations commute - thus (3.7) obviously preserves the constant curvature property. In a sense, one should regard both $x_{2}$ and $t$ as time-like variables. A similar instance of time-like evolution in spatial directions was reported in [2]. It is not known whether more complicated evolutions (e.g. $\left.U=\omega_{, 1}+\omega_{, 2}\right)$ can give rise to integrable equations.

Now that it is known that this surface motion can be expressed as the motion of a space curve it is fruitful to explore further. The coordinate lines are asymptotic and hence their principal unit normals lie in the surface (this can be seen from (3.3), because $T_{1,1}$ is proportional to the principal unit normal of the $x_{1}$-curve). Furthermore for the particles in a coordinate line to trace out the coordinate grid properly it is easy to see (see Figure 2) that

$$
\mathbf{r}_{, 2}=\sin \omega \mathbf{n}_{c}+\cos \omega \mathbf{t}_{c} \equiv U_{c} \mathbf{n}_{c}+W_{c} \mathbf{t}_{c}
$$

where the subscript ${ }_{c}$ denotes properties of an $x_{1}$-coordinate curve, $\kappa_{c}$ is the curvature of the coordinate curve, and $\tau_{c}$ is its torsion. We know that this "motion" (i.e., motion in the $x_{2}$-direction, as the curve sweeps out the initial surface) maintains the arc-length parameterization, which requires $W_{, 1}=\kappa_{c} U \Rightarrow$ $\kappa_{c}=-\omega_{, 1}$. Now take this curve motion and find $\kappa_{c, 2}$ using the space-curve evolution equations of [14]: the result is

$$
\kappa_{c, 2}=-\tau^{2} \sin \omega
$$

Because $\omega$ satisfies the sine-Gordon equation, we get $\tau=\sqrt{-K}$, i.e., Tchebyshev coordinate lines in a surface of constant negative curvature have constant torsion [5]. The curve is evolving under the equation of motion

$$
\mathbf{r}_{, 2}=-\sin \int \kappa_{c} d s \mathbf{n}_{c}+\cos \int \kappa_{c} d s \mathbf{t}_{c}
$$

We have in fact recovered the constant-torsion sine-Gordon curve equation of Lamb [12]. However, due to his inverse method (specifying $\dot{\mathbf{t}}, \dot{\mathbf{n}}, \dot{\mathbf{b}}$ instead of $\dot{\mathbf{r}}$ ), he was unable to determine $\dot{\mathbf{r}}$ for this evolution. In the forward direction it is easy to check that we have in fact the same evolution.

(NOTE: In the literature there are two distinct curve motions which obey the sine-Gordon equation. Let the curvature evolve by $\dot{\kappa}_{c} \equiv \Omega U$. Lamb's evolution, given by (3.8), has $\Omega U=0$, and the curve would not move at all if $\tau_{c}=0$. Nakayama et al. [14] found a planar curve motion which obeys sine-Gordon: it has $\Omega^{2} U=0$.)

As pointed out above, we can also think of an $x_{1}$-coordinate line as moving in the $t$-direction, governed by the equation of motion (see Figure 2)

$$
\begin{aligned}
\dot{\mathbf{r}} & =U \mathbf{b}_{c}+V^{1} \mathbf{t}_{c}+V^{2}\left(\cos \omega \mathbf{t}_{c}+\sin \omega \mathbf{n}_{c}\right) \\
& =-\kappa_{c} \mathbf{b}_{c}+\kappa_{c, 1} \mathbf{n}_{c} / 2 \sqrt{-K}+\kappa_{c}^{2} \mathbf{t}_{c} / 4 \sqrt{-K}
\end{aligned}
$$


The final cross-check is that a space curve with motion given by (3.10) has (from the curve equations in Nakayama et al. [14]) intrinsic evolution

$$
\begin{aligned}
\dot{\kappa}_{c} & =\frac{3}{2} \sqrt{-K} \kappa_{c, s}+\left(3 \kappa_{c}^{2} \kappa_{c, s}+2 \kappa_{c, s s s}\right) / 4 \sqrt{-K} \\
\dot{\tau}_{c} & =0
\end{aligned}
$$

agreeing with (3.7) when $\kappa_{c}=-\omega_{, 1}$.

Acknowledgements: The authors are grateful for helpful conversations with S. Chakravarty, M. Mineev, K. Nakayama, and M. Wadati, and to Nakayama and Wadati for sharing their manuscript prior to publication. This work was supported in part by NSF grant \#DMS9096156.

\section{REFERENCES}

1. M. J. Ablowitz, D. J. Kaup, A. C. Newell, and H. Segur, The inverse scattering transformFourier analysis for nonlinear problems, Stud. Appl. Math. 53 (1974), 249-315.

2. M. J. Ablowitz, R. Beals, and K. Tenenblat, On the solution of the generalized wave and generalized sine-Gordon equations, Stud. Appl. Math. 74 (1986), 177-203; Addenda and corrigenda, 77 (1987) 101.

3. R. Brower, D. Kessler, J. Koplik, and H. Levine, Geometrical models of interface evolution, Phys. Rev. A 29 (1984), 1335-1342.

4. J. D. Buckmaster and G. S. S. Ludford, Lectures on Mathematical Combustion, CBMSNSF Series in Applied Math., vol. 43, SIAM, Philadelphia, 1983.

5. L. P. Eisenhart, An Introduction to Differential Geometry, Princeton University Press, Princeton, 1947.

6. M. Gage and R. S. Hamilton, The heat equation shrinking convex plane curves, J. Diff. Geom. 23 (1986), 69-96.

7. R. E. Goldstein and D. M. Petrich, The Korteweg-de Vries hierarchy as dynamics of closed curves in the plane, Phys. Rev. Lett. 67 (1991), 3203-3206.

8. M. Grayson, The heat equations shrinks embedded plane curves to round points, J. Diff. Geom. 26 (1987), 285-314.

9. Hasimoto, A soliton on a vortex filament, J. Fluid Mech. 51 (1972), 477-485.

10. G. Huisken, Flow by mean curvature of convex surfaces into spheres, J. Diff. Geom. 20 (1984), 237-266.

11. D. A. Kessler, J. Koplik, and H. Levine, Pattern selection in fingered growth phenomena, Adv. Phys. 37 (1988), 255-339.

12. G. L. Lamb, Jr., Solitons on moving space curves, J. Math. Phys. 18 (1977), 1654-1661.

13. J. S. Langer, Lectures in the theory of pattern formation, in Chance and Matter (J. Soutelie, J. Vannimenus, and R. Stora, eds.), North-Holland, New York, 1987, pp. 629-712.

14. K. Nakayama, H. Segur, and M. Wadati, Integrability and the motion of curves, Phys. Rev. Lett. 69 (1992), 2603-2606.

15. K. Nakayama and M. Wadati, The motion of surfaces, preprint (1992).

16. P. Pelcé, Dynamics of Curved Fronts, Academic Press, New York, 1988.

17. J. A. Sethian, Numerical algorithms for propagating interfaces: Hamilton-Jacobi equations and conservation laws, J. Diff. Geom. 31 (1990), 131-161.

18. J. Smoller, Schock Waves and Reaction-Diffusion Equations, Springer, New York, 1980.

19. M. Spivak, A Comprehensive Introduction to Differential Geometry, Volume III, Publish or Perish, Inc., Boston, 1975.

20. J. J. Stoker, Water Waves, Wiley-Interscience, New York, 1957. 
Figure 1. Definition of the metric.

An origin is fixed and two coordinate lines are chosen to be parameterized by arclength for all time; the metric at other points such as $p$ is found by integrating the Gauss-Codazzi equations along a coordinate line.

FIgURE 2. Tchebyshev net on a surface of constant negative curvature.

All vectors shown are unit vectors, the normal $\mathbf{n}_{c}$ to the coordinate curve lies in the surface, and the surface normal $\mathbf{n}$ is at right angles to it.

Program in Applied Mathematics, University of Colorado at Boulder, Boulder, CO 80309-0526

E-mail address: rxm@boulder.colorado.edu, segur@boulder.colorado.edu 